\title{
Molecular and genetic aspects of ethanol in human diet: a nutrient or a toxicant?
}

\author{
Giovambattista Pani · Osvaldo Raùl Koch • \\ Tommaso Galeotti
}

Received: 8 January 2010/Accepted: 11 January 2010/Published online: 20 February 2010

(C) Springer-Verlag 2010

You do not need to be a long-trained toxicologist to know that the very same chemical compound can be harmless or even beneficial to the body under some circumstances, and definitely toxic under others. Ancient Greeks used the same word, "Pharmakon", to indicate both a drug and a poison.

But when it comes to ethanol, acknowledgement of this simple, common sense truth requires a slightly harder effort.

Ethanol has been for thousands of years an integral component of human nutrition (Fig. 1), with an impact on culture, arts, social relationships and maybe Nations' fates very difficult to quantify. Ethyl alcohol is an aliment with an energy content higher than sugar and proteins and close to lipids and may account for overall $5 \%$ of daily caloric intake in many Western countries [1]. Additionally, low to moderate ethanol consumption has been shown to significantly decrease cardiovascular risk and mortality rate, an effect distinct from that of polyphenols present in wine together with alcohol. Yet, alcohol abuse has a terrible impact on human health because of alcoholic liver disease, cerebrovascular disorders, dysmetabolism, malnutrition and cancer. Although in principle any kind of food may result harmful when assumed in excess amount, for hardly any other constituent of human diet consequences on health can be so dramatically diverse and the relative critical "doses" so dangerously close.

Alcohol: nutrient or toxicant? Toxicologists have no doubts about: both, is the answer, depending on the

G. Pani $(\bowtie) \cdot$ T. Galeotti

Institute of General Pathology,

Catholic University Medical School, 00168 Rome, Italy

e-mail: gpani@rm.unicatt.it

O. R. Koch

Department of Pathology, Faculty of Medicine University

of Buenos Aires, Buenos Aires, Argentina amount assumed. One may try to be slightly more precise and stress that not only "how much" but also "how often" or "how regularly" you drink may make the difference. But in the age of "omics", these important answers are likely to be still insufficient. Individual (or group) predisposition to disease is written in the genes and in the proteins, and high-throughput investigation into human genome and proteome is beginning to reveal in its complexity the genetic component of many (the most important) multifactorial diseases. Along similar lines, pharmacogenomics is actively searching for the factors dictating individual responses to drugs, an avenue to tomorrow "personalized" therapies.

Time has come to import the same logic into alcohol research. It is long known that race and sex-related differences exist in relation to alcohol metabolism and that this definitely impacts on ethanol adverse effects (see Seitz et al., in this issue); do similar differences operate also at an individual level? Could these genetic differences contribute in part to make alcohol a toxic from a nutrient or the other way around? More important, at which cellular and molecular level do these differences operate? The more information we get from high-throughput, genome-scale analyses on genetic associations between ethanol and disease, the more we need to structure this information into a biochemical context. And association of genetic traits with ethanol toxicity may not always be so straightforward as for those involving, for instance, ethanol-catabolizing enzymes [2] or determinants of the intracellular redox balance. Hence, more knowledge on the molecular mechanisms and biochemical circuitries underlying ethanol adverse (or beneficial) effects is critically needed.

The present issue of Genes and Nutrition offers a unique overview of the most up-to-date molecular and genetic aspects of ethanol as a component of human diet. 


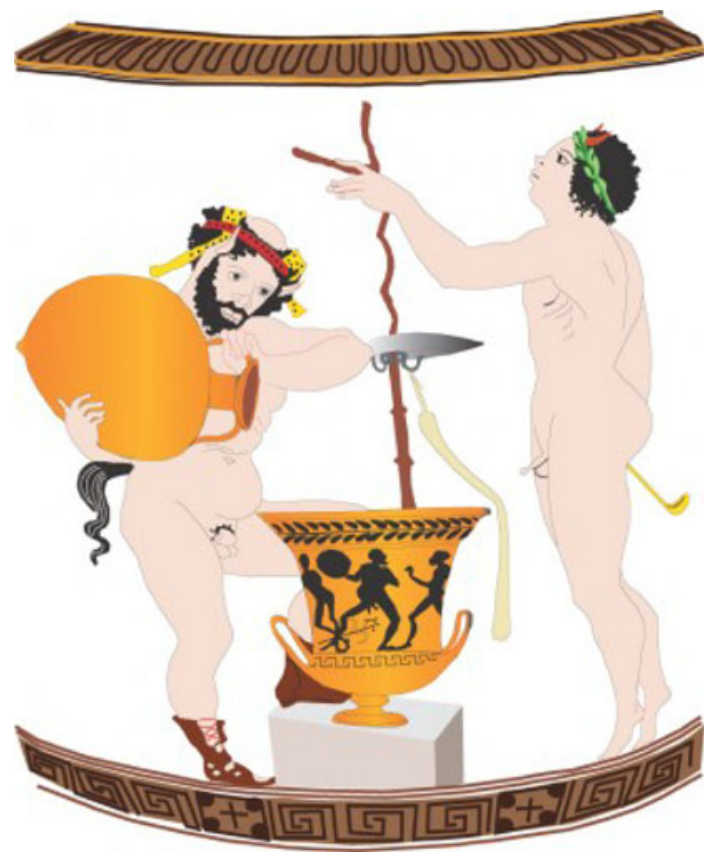

Fig. 1 Satyr and Faun; A satyr and a faun are preparing the mixture of wine and water

Shepard et al. underscore how, at a global gene expression level, chronic and acute, "binge" ethanol consumption trigger distinct gene expression programs, operating through different mechanism of epigenetic control at the chromatin level. Thus, profound differences exist in cell responses to these two modality of exposure to ethanol.

Comporti et al. (doi:10.1007/s12263-009-0159-9) offer an exhaustive overview of the main mechanisms of cellular damage by alcohol, with special attention to redox reactions involving Cyp2E1 and lipid peroxidation. Oxidative stress as a key mediator of ethanol toxicity recurs, but under the special angle of inflammation and immunity, in two other important contributions. Cederbaum et al. summarize recent evidence on the cooperation, in the establishment of alcohol-related liver damage, between the Cyp2E1 system in hepatocytes and ethanol-induced generation of ROS and cytokines by Kupffer cells. In a different perspective, Albano et al. describe how oxygen species produced by alcohol may trigger autoimmune reactions against oxidized liver antigens (like Cyp2E1 itself), thereby explaining many inflammatory and immunological aspects of ALD pathology.

Do redox reactions also underlie "beneficial" effects of ethanol? Lakshman et al. (page) focus on induction of the HDL component paraoxonase 1 (PON-1), endowed of antioxidant activity on LDL, as a major antiatherogenic effect of moderate alcohol. Interestingly, the same enzyme results inhibited in heavy drinkers, a condition associated, as opposite to moderate alcohol consumption, with increased risk for coronary artery disease.

On the other hand, additional mechanism of ethanol action is emerging (see Shepard et al., doi:10.1007/s12263009-0156-z), like effects on intracellular trafficking, which does not appear to directly involve generation of ROS, but rather acetaldehyde as a direct ethanol catabolite. Acetaldehyde accumulation plays also a major role in mutagenesis and tumorigenesis by ethanol, and, importantly, polymorphisms or mutations of genes coding for AA generation or detoxifying enzymes, resulting in elevated AA concentrations, are associated with increased cancer risk (Seitz et al. page).

As we know more and more on molecules and genes underlying ethanol actions as nutrient or toxicant, new perspectives open up and even more questions arise.

To which extent newly discovered molecular determinants of cell responses to ethanol are polymorphic within human populations? Is there a "genetic" signature we need to uncover, which predicts how an individual responds to ethanol? How important are inflammation and immunity for alcohol-mediated cell damage? Could alcohol-related diseases and inflammatory/ autoimmune diseases share some aspects of genetic predisposition?

While trying to answer these questions, we should also keep in mind that reactive oxygen species not only damage cells but also operate physiologically as signal transduction molecules [3]. Ethanol engages important signal transduction machineries $[4,5]$ and also operates as a fine regulator of gene expression. Should we start to consider alcohol-related diseases as "signaling diseases"? How much information, already available on signaling deregulation in cancer, inflammation or neurodegeneration, can be exploited to understand more about ethanol effects on human cell? Finally, could the difference between a "weak" and a "strong", a transient or a persistent intracellular signal, be what makes the difference between ethanol as a nutrient, a beneficial drug, or a poison?

Many questions, a long way ahead....

\section{References}

1. Santolaria F, Pérez-Manzano JL, Milena A, González-Reimers E, Gómez-Rodríguez MA, Martínez-Riera A, Alemán-Valls MR, de la Vega-Prieto MJ (2000) Nutritional assessment in alcoholic patients. Its relationship with alcoholic intake, feeding habits, organic complications and social problems. Drug Alcohol Depend 59(3):295-304

2. Ingelman-Sundberg M, Johansson I, Yin H, Terelius Y, Eliasson E, Clot P, Albano E (1993) Ethanol-inducible cytochrome P4502E1: 
genetic polymorphism, regulation, and possible role in the etiology of alcohol-induced liver disease. Alcohol 10(6):447-452

3. Finkel T (2001) Reactive oxygen species and signal transduction. IUBMB Life 52(1-2):3-6

4. Koch OR, Fusco S, Ranieri SC, Maulucci G, Palozza P, Larocca LM, Cravero AA, Farre' SM, De Spirito M, Galeotti T, Pani G
(2008) Role of the life span determinant P66(shcA) in ethanolinduced liver damage. Lab Invest 88(7):750-760

5. Dey A, Cederbaum AI (2006) Alcohol and oxidative liver injury. Hepatology 43(2 Suppl 1):S63-S74 